\title{
Macrophage Imaging of Intracranial Aneurysms
}

\author{
Kampei SHIMIZU, ${ }^{1,2}$ Mika KUSHAMAE, ${ }^{1,3}$ and Tomohiro AOKI ${ }^{1}$ \\ ${ }^{1}$ Department of Molecular Pharmacology, Research Institute, \\ National Cerebral and Cardiovascular Center, Suita, Osaka, Japan; \\ ${ }^{2}$ Department of Neurosurgery, Kyoto University Graduate \\ School of Medicine, Kyoto, Kyoto, Japan; \\ ${ }^{3}$ Department of Neurosurgery, Showa University \\ School of Medicine, Tokyo, Japan
}

\begin{abstract}
Considered with a poor outcome of subarachnoid hemorrhage due to rupture of intracranial aneurysms (IAs), treatment interventions to prevent rupture of the lesions are mandatory for social health. As treatment option is limited to surgical manipulations, like microsurgical clipping, endovascular coiling or deployment of flow diverter, and these surgical interventions have a potential risk of complications in nature, a proper selection of rupture-prone IAs among ones incidentally found is essential. Today, a rupture risk in each case is estimated by several factors like patient characteristics and morphological ones of each lesion. However, unfortunately, an IA without treatment sometimes unexpectedly ruptures resulting in a devastating outcome or an IA surgically treated is turned out to have a thick wall. To achieve more efficient treatment interventions, the development of a novel diagnostic modality is required. Here, mainly through the accumulation of experimental findings, the crucial contribution of macrophagemediated chronic inflammatory responses to IA progression have been revealed, making macrophage being a promising target for a diagnosis. If we could non-invasively visualize accumulation of macrophages in lesions, this imaging technique 'macrophage imaging' may enable a qualitative evaluation of IAs to stratify rupture-prone 'dangerous' lesions among many stable ones. Thereby, a development of macrophage imaging makes an indication of surgical interventions being more accurate and also greatly facilitates a development of a novel medical therapy if used as a surrogate marker.
\end{abstract}

Key words: intracranial aneurysm, macrophage, imaging, ferumoxytol, chronic inflammation

\section{Introduction}

Unruptured intracranial aneurysm (IA) is a common disease found in about $2-5 \%$ of general population. ${ }^{1)}$ Unruptured IAs are incidentally found during brain check or so especially in Japan. Patients with such incidentally found lesions have sometimes been treated preemptively because of the high morbidity and mortality of subarachnoid hemorrhage due to rupture of pre-existing IAs. ${ }^{2)}$ Current available options to achieve the preemptive medicine preventing rupture of IAs are limited to surgical interventions (i.e. microsurgery or endovascular surgery). Indications of surgical interventions as the pre-emptive medicine have been determined on a case-to-case

Received February 13, 2019; Accepted March 25, 2019

Copyright $\odot 2019$ by The Japan Neurosurgical Society This work is licensed under a Creative Commons AttributionNonCommercial-NoDerivatives International License. basis through carefully considering estimated annual rupture rates of each lesion and potential risks posed by surgical interventions. ${ }^{3,4}$ Annual rupture rates of each IA lesion are currently estimated ${ }^{3)}$ through combination of some predictors indicated by prospective human cohort studies, ${ }^{5-10)}$ e.g. a size, a shape or a location as aneurysmal factors and a race, an age or confounding diseases. In another point of view, because complications by surgical intervention are not negligible in nature, a development of novel predictors of rupture for each lesion makes indications of surgical interventions more appropriate.

Recent experimental findings has clarified the role of macrophage-mediated chronic inflammation in the promotion of the pathogenesis of IAs. ${ }^{11-16)}$ A development of an imaging technique visualizing macrophages infiltrating in lesions, so called 'macrophage imaging', and an evaluation of its usefulness as a surrogate to predict progression and rupture of lesions has thus become of great interest. Macrophage imaging may 
be able to detect a qualitatively different aspect of each IA lesion from well-established predictors of rupture, ${ }^{5-8,10)}$ and thereby provide more direct reference regarding inflammatory status, 'activity', of individual lesions.

To date, several studies have already demonstrated the potential of macrophage imaging as a surrogate marker to stratify rupture-prone IAs. ${ }^{17-19)}$ In this review article, we have summarized the biological rationale, technical aspects, the usefulness, and the future perspective of macrophage imaging as a potential diagnostic tool of IAs.

\section{Macrophage Infiltration as a Surrogate Marker to Predict the Progression of IAs}

Macrophage imaging is a diagnostic tool utilizing phagocytic activity of macrophages. The presence of macrophages in tissues or lesions can be monitored through visualizing contrast agents engulfed by them. The potential of this imaging technique as a diagnostic tool to visualize macrophage-mediated inflammatory responses in situ has already been indicated in several diseases like atherosclerosis, cancers, and inflammation in the central nervous system. ${ }^{18,20-24)}$ In this section, we have summarized the previous experimental findings and a rationale to use the presence of macrophages as a surrogate marker to predict the progression or rupture of IAs.

Histopathological analyses of surgically resected human IA specimens have revealed that the number of macrophages present in lesions is larger in ruptured IAs than that in unruptured ones. ${ }^{25,26)}$ Because degenerative changes in IA walls (e.g. an endothelial injury or a loss of medial smooth muscle cells) are prominent features of ruptured IAs, ${ }^{25,26)}$ many believe the involvement of macrophages infiltrating to lesions in the progression of IAs.

The crucial role of macrophages in the pathogenesis of IAs has been further investigated in animal studies and a basic concept has been clarified. Macrophages can be observed in IA lesions induced in animal models of $\mathrm{IAs}^{27,28)}$ as a type of cells whose number is largest among inflammatory cells. ${ }^{13)}$ The crucial contribution of macrophages to the progression of the disease has been supported by some experimental findings. For example, an inhibition of monocyte chemotactic protein-1 (MCP-1), a cardinal chemoattractant protein for macrophages, or a genetic deletion of CCL2 (which encodes MCP-1), significantly suppresses infiltration of macrophages in lesions and also the progression of the lesion. ${ }^{12)} \mathrm{A}$ pharmacological depletion of macrophages by Clodronate liposome consistently and significantly inhibits the development of IAs. ${ }^{16)}$ Although macrophages not only evoke inflammatory responses but also function to repair damaged tissues and thus the role of macrophages in vascular inflammation are complex and ununiform, ${ }^{29,30)}$ above findings suggest that macrophages infiltrating to IA lesions as a whole facilitate the pathophysiology of IAs. This assumption is in line with a histopathological investigation of surgically resected human IA specimens. ${ }^{31)}$ In this study, both the two major subpopulation of macrophages, $\mathrm{M}_{1}$ lineage with pro-inflammatory actions and $\mathrm{M}_{2}$ lineage with anti-inflammatory actions, could be observed in IA walls before rupture, while $\mathrm{M}_{1}$ linage becomes predominant after rupture. ${ }^{31)}$ Not only the precise spatiotemporal changes of each subpopulation of macrophages in IA lesion during the disease progression but also the contribution of each subpopulation in the pathogenesis, however, remains to be elucidated.

A genetic deletion of p50 subunit of nuclear factor $\kappa \mathrm{B}(\mathrm{NF}-\kappa \mathrm{B})$ or a pharmacological inhibition of NF- $\kappa \mathrm{B}$ activity significantly suppresses the development of IAs. ${ }^{32)}$ Because a transcription factor NF- $\kappa$ B plays a central role in regulating (in most cases, triggering) inflammatory responses via inducing various proinflammatory genes as a major transcription factor for them, these findings suggest that IA is one of the inflammation-mediated diseases. Indeed, in above experiments deleting or inhibiting NF- $\kappa \mathrm{B}$, expressions of pro-inflammatory genes like IL- $1 \beta$, cyclooxygenase- 2 (COX-2) and inducible nitric oxide synthese (iNOS) in lesions are remarkably suppressed. ${ }^{32)}$ Furthermore, because a genetic deletion or a pharmacological inhibition of pro-inflammatory molecules downstream of NF- $\kappa \mathrm{B}$, including TNF- $\alpha,{ }^{33,34)} \mathrm{IL}-1 \beta{ }^{35)}$ cyclooxygenase-2 (COX-2), ${ }^{36)}$ inducible nitric oxide synthese (iNOS), ${ }^{37)}$ and matrix metalloproteinase-9 (MMP-9) matrix metalloproteinase-9 (MMP-9), ${ }^{13,38)}$ consistently and significantly suppresses the progression of IAs, $\mathrm{NF}-\kappa \mathrm{B}$-mediated inflammatory responses are supposed to regulate the pathogenesis of IAs.

In experiments using NF- $\kappa \mathrm{B}$ reporter mice in which $\mathrm{NF}-\kappa \mathrm{B}$ activation can be monitored by an expression of a fluorescent protein, NF- $\kappa \mathrm{B}$ first activates in endothelial cells and macrophages infiltrating in adventitia at the early stage of IA formation. ${ }^{11)}$ Through a cell type-specific inhibition of $\mathrm{NF}-\kappa \mathrm{B}$ activation by expressing the mutated form of $\mathrm{I} \kappa \mathrm{B} \alpha$ under the control of Cre-LoxP system, the crucial role of NF- $\kappa \mathrm{B}$ activation in macrophages in the pathogenesis of IAs is revealed. ${ }^{11)}$ Indeed, MMP-2 and nine mainly produced by macrophages in the NF- $\kappa \mathrm{B}$-dependent manner promote the initiation and progression of the disease. ${ }^{13,32)}$ Cysteine cathepsins, which are extracellular matrix-degrading proteinase like MMPs, are also induced in various cell types including macrophages in IA lesions, and induces fragility 
of the walls. ${ }^{39)}$ These findings support the crucial role of macrophages in degradation of extracellular matrix in situ to exacerbate the fragility of IA wall. Recently, prostaglandin E receptor subtype 2 (EP2) cascade is identified as an upstream signal cascade to activate NF- $\kappa \mathrm{B}$ in lesions. ${ }^{11,36)}$ As a macrophagespecific deletion of Ptger2 (which encodes EP2) can suppress the development of IAs ${ }^{11)}$ consistently with results about NF- $\kappa \mathrm{B}$, the importance of macrophages and inflammatory cascade functioning there in the pathogenesis has been further supported. Here importantly, the prostaglandin E (PGE)2-EP2-NF- $\kappa$ B signaling cascade in macrophages functions not only to initiate but also to sustenance of inflammatory responses in situ once triggered through forming a positive feedback loop via inducing COX-2. In addition, this signaling cascade in macrophages synergizes with a cardinal pro-inflammatory cytokine, TNF- $\alpha$, present in situ ${ }^{33,34)}$ to amplify inflammatory response there. ${ }^{11)}$ Also, this cascade enhances MCP-1 expression and contributes to the formation of auto-amplification among macrophages, making the macrophage-mediated inflammatory responses in lesions being exacerbated. ${ }^{11)}$

Macrophage-mediated chronic inflammation has thus been identified as one of the essential factors in the progression of IAs. Therefore, visualizing macrophages in IA lesions can become a reasonable strategy to predict the progression of lesions.

\section{Macrophage Imaging}

As discussed in the previous section, macrophage imaging may enable to evaluate inflammation status in IA lesions ${ }^{17,40)}$ (Fig. 1).

Ferumoxytol is an ultrasmall superparamagnetic iron oxide particle (USPIO) used for the treatment of anemia due to chronic renal diseases. As macrophages engulf USPIO (Fig. 2A) and this particle exerts strong $\mathrm{T}_{2}^{*}$ effect in MRI, ${ }^{41)}$ USPIO can also be used as a contrast agent for detecting macrophages in MRI. Indeed, macrophage imaging using USPIO has been demonstrated in oncology and inflammation in the central nervous system. ${ }^{18,20-24)}$ Ferumoxytol has $30 \mathrm{~nm}$ in the hydrodynamic diameter and its circulating half-life is about $14 \mathrm{~h}$ in human. Because the diameter of ferumoxytol is remarkably smaller than well-used gadolinium-containing contrast agent with the diameter $1 \mathrm{~nm}$ and is thus retained much longer in the blood, ferumoxytol disappears much more slowly from blood stream but may cross the impaired blood-brain barrier more specifically
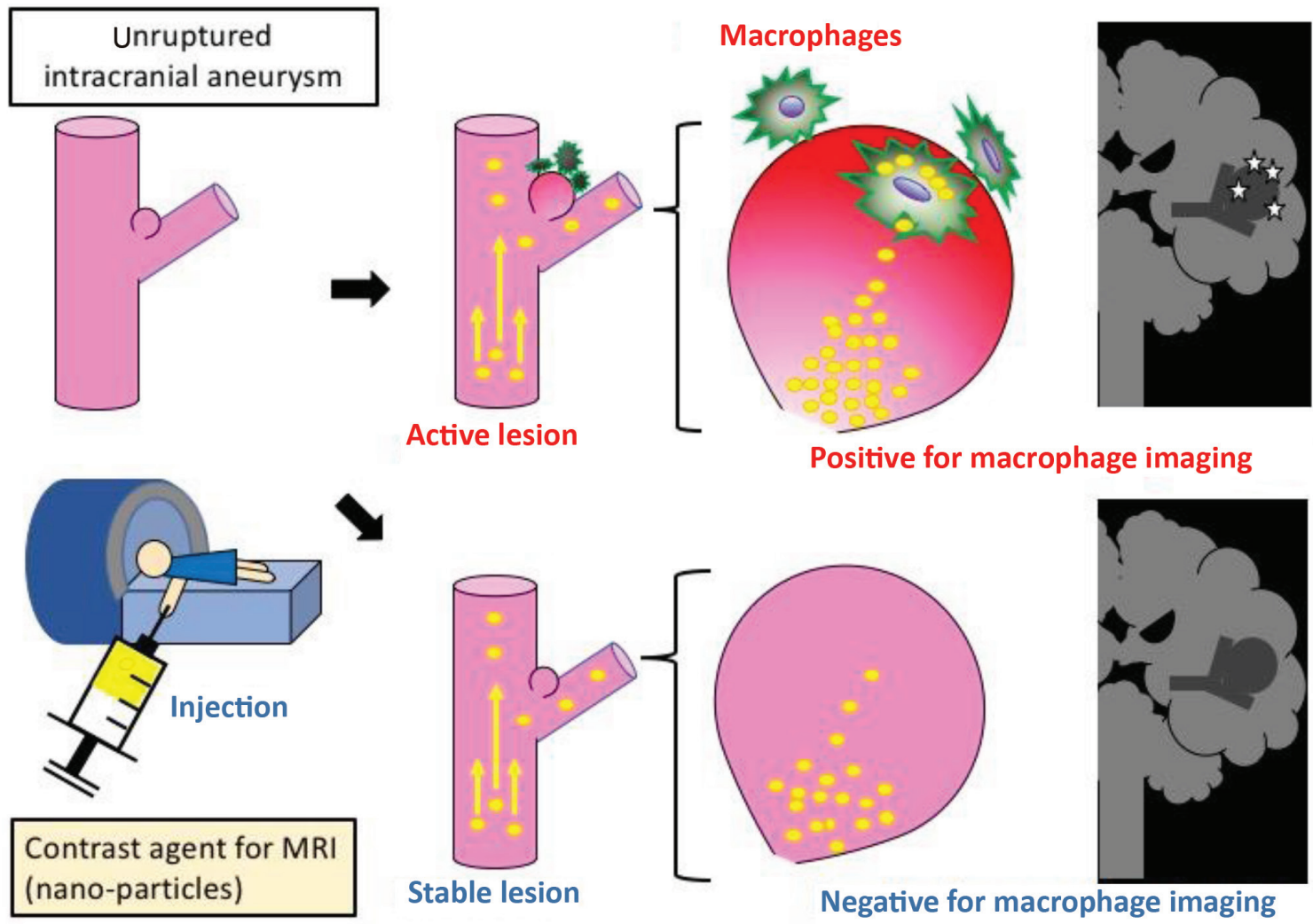

Positive for macrophage imaging

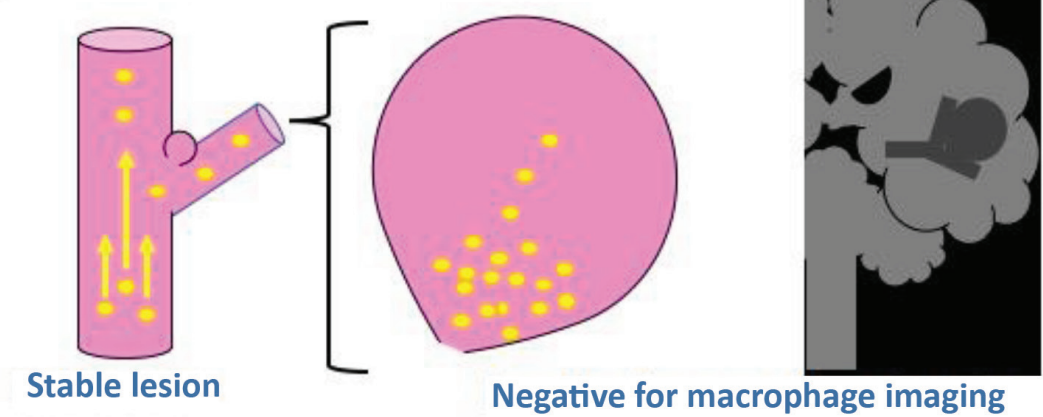

Fig. 1 The schematic drawing of the concept of macrophage imaging. Nano-particles are injected to a patient with an intracranial aneurysm and macrophages in a lesion engulfing these particles are visualized by MRI. The lesion with enhancement is stratified as an 'active' one. 


\section{A Ferumoxytol treatment}

$(-)$

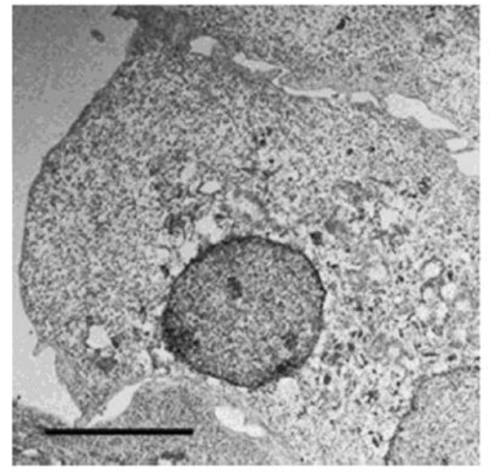

B

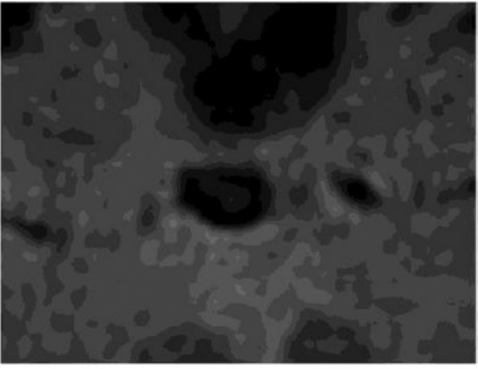

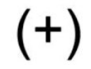

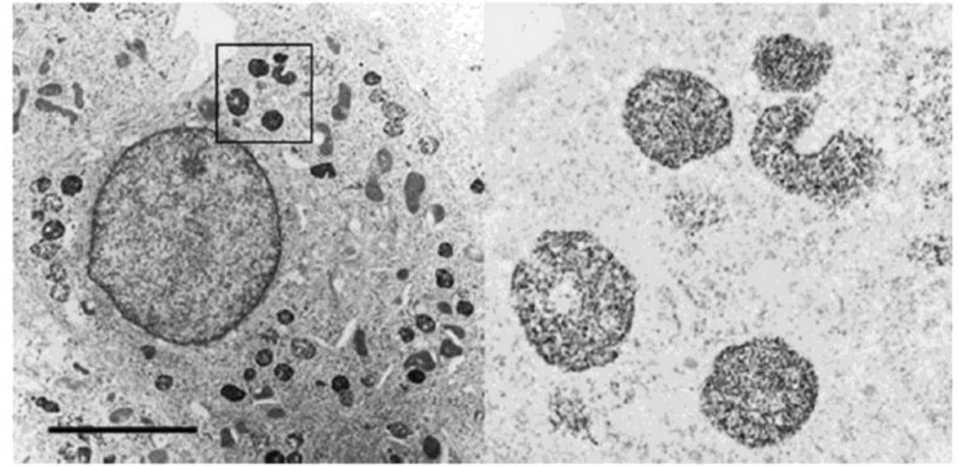

Post

Subtracted image
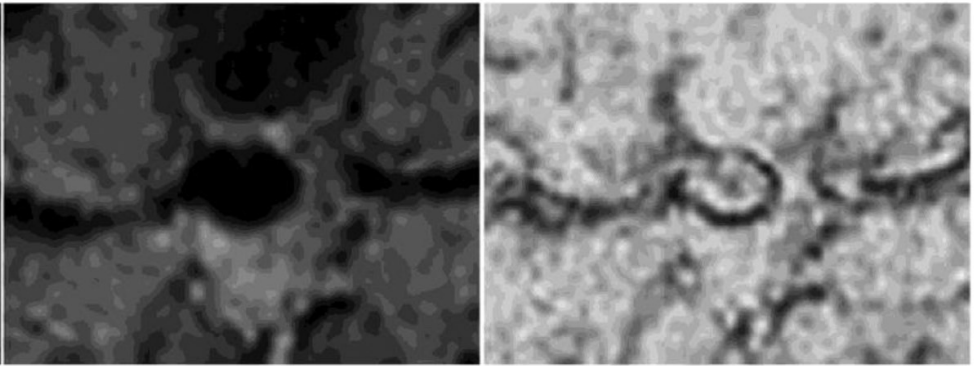

Fig. 2 Macrophage imaging by an iron-containing nanoparticle, ferumoxytol. (A) Electron microscopic imaging of RAW264.7 cells, a mouse macrophage cell line, engulfing ferumoxytol. Cultured Raw264.7 cells were treated with ferumoxytol and subjected to an electron microscopic observation. Magnified image of RAW264.7 cells engulfing ferumoxytol is shown in the right panel. (B) One example of macrophage imaging of human case with a cavernous aneurysm by ferumoxytol. 2D-Gradient echo MR images were acquired before (pre) and $24 \mathrm{~h}$ after (post) the administration of macrophage imaging. Subtracted image is shown in the right panel. Note the presence of several hypo-intensity signals in the aneurysm wall. Images in this figure were originally published in citation $^{17}$ as Figs. $2 \mathrm{~A}$ and $4 \mathrm{~A}$ (B). Written permission regarding the reuse of these images in this figure was acquired.

in damaged tissues resulting in the uptake by macrophages infiltrating there. The previous report about inflammation in the central nervous system has demonstrated that the enhancement of lesions begins within several hours after injection, forms peak at around $24 \mathrm{~h}$ and lasts at least for 3 days. ${ }^{42)}$ Consistently, in the previous studies dealing IAs, enhancement of IA walls by ferumoxytol is well demonstrated at $24-72 \mathrm{~h}$ after administration ${ }^{17,40)}$. Note that the uptake of ferumoxytol in macrophages infiltrating in IA lesions is confirmed by a Berlin blue staining at $24-72 \mathrm{~h}$ in human IA specimens. ${ }^{40)}$ Importantly, enhancement of IA walls by ferumoxytol at $24 \mathrm{~h}$ after administration is associated with rupture of the lesion within 6 months after the imaging, ${ }^{18)}$ supporting the notion that macrophage imaging can predict rupture of lesions. Here, intriguingly, oral intake of aspirin ( $81 \mathrm{mg} /$ day) with anti-inflammatory effect attenuates the enhancement of IA walls by ferumoxytol in 3 months. ${ }^{19)}$ These findings suggest that macrophage imaging is useful not only for monitoring macrophage-mediated inflammation in situ but also for evaluating the efficacy of medication when used as a surrogate maker.

There are, however, several limitations in the usage of ferumoxytol as a contrast agent for macrophage imaging to predict rupture-prone IAs. First, the indication of ferumoxytol is strictly limited to iron deficiency anemia due to chronic kidney disease by the Food and Drug Administration because of its potential risk of a lethal allergic reaction. Second, because iron is abundantly contained in red blood cells in the blood stream as a component of hemoglobin, subtraction of images between pre- and postinjection to discriminate signals from hemoglobin is required for detecting the presence of iron in macrophages in IA walls (Fig. 2B). Considered the resolution of MRI and the thickness of IA walls, 
the subtraction of two independently acquired MRI $\mathrm{T}_{2}^{*}$ images is quite challenging and time-consuming, making the macrophage imaging by ferumoxytol being technically difficult and the usage of this imaging technique in a clinical setting being almost impossible. Another limitation is associated with an optimal dose; administration of ferumoxytol at a high dose is advantageous in visualizing its presence in tissues, but the signal-to-noise ratio is inversely correlated with the dose. ${ }^{43}$

The potential of another vessel wall imaging using conventional gadolinium contrast agents as a diagnostic tool has also been reported. ${ }^{44-46)}$ In these studies, the circumferential enhancement of human IA walls by conventional $\mathrm{T}_{1}$-weighted imaging with gadolinium contrast agents is correlated with the progression of lesions. ${ }^{44-46)}$ Histopathological examinations of these IA specimens with enhancement have revealed the association of vessel wall enhancement with the presence of vasa vasorum formations and abundant macrophage infiltrations. ${ }^{47,48)}$ Therefore, the enhancement with contrast agents in MRI might reflect the inflammation in lesions, although mechanisms underlying enhancements have not been revealed. Nonetheless, these studies imply that the accumulation of gadolinium even in thin IA walls can be visualized in MRI. Considering these findings and above limitations about USPIO, positive contrast agents like gadolinium-containing nanoparticles, may thus be more suitable as a contrast agent used for macrophage imaging. The development of a novel contrast agent for macrophage imaging applicable in a clinical setting is demanded to achieve more efficient preemptive treatment for IAs.

\section{Conflicts of Interest Disclosure}

All authors have no conflicts of interest and registered online Self-reported COI Disclosure Statement Forms through the website for The Japan Neurosurgical Society.

\section{References}

1) Vlak MH, Algra A, Brandenburg R, Rinkel GJ: Prevalence of unruptured intracranial aneurysms, with emphasis on sex, age, comorbidity, country, and time period: a systematic review and meta-analysis. Lancet Neurol 10: 626-636, 2011

2) Aoki T, Nozaki K: Preemptive medicine for cerebral aneurysms. Neurol Med Chir (Tokyo) 56: 552-568, 2016

3) Greving JP, Wermer MJ, Brown RD, et al.: Development of the PHASES score for prediction of risk of rupture of intracranial aneurysms: a pooled analysis of six prospective cohort studies. Lancet Neurol 13: 59-66, 2014

4) Kotowski M, Naggara O, Darsaut TE, et al.: Safety and occlusion rates of surgical treatment of unruptured intracranial aneurysms: a systematic review and meta-analysis of the literature from 1990 to 2011. J Neurol Neurosurg Psychiatry 84: 42-48, 2013

5) Ishibashi T, Murayama Y, Urashima M, et al.: Unruptured intracranial aneurysms: incidence of rupture and risk factors. Stroke 40: 313-316, 2009

6) Juvela S, Porras M, Poussa K: Natural history of unruptured intracranial aneurysms: probability of and risk factors for aneurysm rupture. J Neurosurg 93: $379-387,2000$

7) UCAS Japan Investigators, Morita A, Kirino T, Hashi K, et al.: The natural course of unruptured cerebral aneurysms in a Japanese cohort. $N$ Engl J Med 366: 2474-2482, 2012

8) Sonobe M, Yamazaki T, Yonekura M, Kikuchi H: Small unruptured intracranial aneurysm verification study: SUAVe study, Japan. Stroke 41: 1969-1977, 2010

9) Wermer MJ, van der Schaaf IC, Velthuis BK, Majoie CB, Albrecht KW, Rinkel GJ: Yield of short-term follow-up CT/MR angiography for small aneurysms detected at screening. Stroke 37: 414-418, 2006

10) Wiebers DO, Whisnant JP, Huston J, et al.: Unruptured intracranial aneurysms: natural history, clinical outcome, and risks of surgical and endovascular treatment. Lancet 362: 103-110, 2003

11) Aoki T, Frôsen J, Fukuda M, et al.: Prostaglandin E2-EP2-NF- $\mathrm{KB}$ signaling in macrophages as a potential therapeutic target for intracranial aneurysms. Sci Signal 10 pii: eaah6037, 2017

12) Aoki T, Kataoka H, Ishibashi R, Nozaki K, Egashira $\mathrm{K}$, Hashimoto N: Impact of monocyte chemoattractant protein-1 deficiency on cerebral aneurysm formation. Stroke 40: 942-951, 2009

13) Aoki T, Kataoka H, Morimoto M, Nozaki K, Hashimoto $\mathrm{N}$ : Macrophage-derived matrix metalloproteinase-2 and -9 promote the progression of cerebral aneurysms in rats. Stroke 38: 162-169, 2007

14) Aoki T, Koseki H, Miyata H, Abekura Y, Shimizu $\mathrm{K}$ : [Intracranial aneurysm as an inflammationrelated disease]. No Shinkei Geka 46: 275-294, 2018 (Japaneae)

15) Shimizu K, Kushamae M, Mizutani T, Aoki T: Intracranial aneurysm as a macrophage-mediated inflammatory disease. Neurol Med Chir (Tokyo) 59: 126-132, 2019

16) Kanematsu Y, Kanematsu M, Kurihara C, et al.: Critical roles of macrophages in the formation of intracranial aneurysm. Stroke 42: 173-178, 2011

17) Aoki T, Saito M, Koseki H, et al.: Macrophage imaging of cerebral aneurysms with ferumoxytol: an exploratory study in an animal model and in patients. J Stroke Cerebrovasc Dis 26: 2055-2064, 2017 
18) Hasan D, Chalouhi N, Jabbour P, et al.: Early change in ferumoxytol-enhanced magnetic resonance imaging signal suggests unstable human cerebral aneurysm: a pilot study. Stroke 43: 3258-3265, 2012

19) Hasan DM, Chalouhi N, Jabbour P, et al.: Evidence that acetylsalicylic acid attenuates inflammation in the walls of human cerebral aneurysms: preliminary results. J Am Heart Assoc 2: e000019, 2013

20) Alam SR, Stirrat C, Richards J, et al.: Vascular and plaque imaging with ultrasmall superparamagnetic particles of iron oxide. J Cardiovasc Magn Reson 17: 83, 2015

21) McConnell HL, Schwartz DL, Richardson BE, Woltjer RL, Muldoon LL, Neuwelt EA: Ferumoxytol nanoparticle uptake in brain during acute neuroinflammation is cell-specific. Nanomedicine 12: 1535-1542, 2016

22) Kirschbaum K, Sonner JK, Zeller MW, et al.: In vivo nanoparticle imaging of innate immune cells can serve as a marker of disease severity in a model of multiple sclerosis. Proc Natl Acad Sci USA 113: 13227-13232, 2016

23) Sadat U, Usman A, Gillard JH: Imaging pathobiology of carotid atherosclerosis with ultrasmall superparamagnetic particles of iron oxide: an update. Curr Opin Cardiol 32: 437-440, 2017

24) Iv M, Samghabadi P, Holdsworth S, et al.: Quantification of macrophages in high-grade gliomas by using ferumoxytol-enhanced MRI: a pilot study. Radiology 290: 198-206, 2019

25) Frösen J, Piippo A, Paetau A, et al.: Remodeling of saccular cerebral artery aneurysm wall is associated with rupture: histological analysis of 24 unruptured and 42 ruptured cases. Stroke 35: 2287-2293, 2004

26) Kataoka K, Taneda M, Asai $T$, Kinoshita A, Ito M, Kuroda R: Structural fragility and inflammatory response of ruptured cerebral aneurysms. A comparative study between ruptured and unruptured cerebral aneurysms. Stroke 30: 1396-1401, 1999

27) Aoki T, Nishimura M: The development and the use of experimental animal models to study the underlying mechanisms of CA formation. J Biomed Biotechnol 2011: 535921, 2011

28) Hashimoto N, Handa H, Hazama F: Experimentally induced cerebral aneurysms in rats. Surg Neurol 10: 3-8, 1978

29) Backes D, Rinkel GJ, Laban KG, Algra A, Vergouwen MD: Patient- and aneurysm-specific risk factors for intracranial aneurysm growth: a systematic review and meta-analysis. Stroke 47: 951-957, 2016

30) Murray PJ, Allen JE, Biswas SK, et al.: Macrophage activation and polarization: nomenclature and experimental guidelines. Immunity 41: 14-20, 2014

31) Hasan D, Chalouhi N, Jabbour P, Hashimoto T: Macrophage imbalance (M1 vs. M2) and upregulation of mast cells in wall of ruptured human cerebral aneurysms: preliminary results. J Neuroinflammation 9: 222, 2012
32) Aoki T, Kataoka H, Shimamura M, et al.: NF-kappaB is a key mediator of cerebral aneurysm formation. Circulation 116: 2830-2840, 2007

33) Aoki T, Fukuda M, Nishimura M, Nozaki K, Narumiya S: Critical role of TNF-alpha-TNFR1 signaling in intracranial aneurysm formation. Acta Neuropathol Commun 2: 34, 2014

34) Starke RM, Chalouhi N, Jabbour PM, et al.: Critical role of TNF- $\alpha$ in cerebral aneurysm formation and progression to rupture. J Neuroinflammation 11: 77,2014

35) Moriwaki T, Takagi Y, Sadamasa N, Aoki T, Nozaki K, Hashimoto N: Impaired progression of cerebral aneurysms in interleukin-1beta-deficient mice. Stroke 37: 900-905, 2006

36) Aoki T, Nishimura M, Matsuoka T, et al.: PGE(2) -EP(2) signalling in endothelium is activated by haemodynamic stress and induces cerebral aneurysm through an amplifying loop via NF-кB. Br J Pharmacol 163: 1237-1249, 2011

37) Fukuda S, Hashimoto N, Naritomi H, et al.: Prevention of rat cerebral aneurysm formation by inhibition of nitric oxide synthase. Circulation 101: 2532-2538, 2000

38) Nuki Y, Tsou TL, Kurihara C, Kanematsu M, Kanematsu Y, Hashimoto T: Elastase-induced intracranial aneurysms in hypertensive mice. Hypertension 54: 1337-1344, 2009

39) Aoki T, Kataoka H, Ishibashi R, Nozaki K, Hashimoto N: Cathepsin B, K, and S are expressed in cerebral aneurysms and promote the progression of cerebral aneurysms. Stroke 39: 2603-2610, 2008

40) Hasan DM, Mahaney KB, Magnotta VA, et al.: Macrophage imaging within human cerebral aneurysms wall using ferumoxytol-enhanced MRI: a pilot study. Arterioscler Thromb Vasc Biol 32: 1032-1038, 2012

41) Dósa E, Tuladhar S, Muldoon LL, Hamilton BE, Rooney WD, Neuwelt EA: MRI using ferumoxytol improves the visualization of central nervous system vascular malformations. Stroke 42: 1581-1588, 2011

42) Weinstein JS, Varallyay CG, Dosa E, et al.: Superparamagnetic iron oxide nanoparticles: diagnostic magnetic resonance imaging and potential therapeutic applications in neurooncology and central nervous system inflammatory pathologies, a review. J Cereb Blood Flow Metab 30: 15-35, 2010

43) Yang M, Christoforidis GA, Figueredo T, Heverhagen JT, Abduljalil A, Knopp MV: Dosage determination of ultrasmall particles of iron oxide for the delineation of microvasculature in the Wistar rat brain. Invest Radiol 40: 655-660, 2005

44) Edjlali M, Guédon A, Ben Hassen W, et al.: Circumferential thick enhancement at vessel wall MRI has high specificity for intracranial aneurysm instability. Radiology 289: 181-187, 2018

45) Edjlali M, Gentric JC, Régent-Rodriguez C, et al.: Does aneurysmal wall enhancement on vessel wall MRI help to distinguish stable from unstable intracranial aneurysms? Stroke 45: 3704-3706, 2014 
46) Omodaka S, Endo H, Niizuma K, et al.: Circumferential wall enhancement in evolving intracranial aneurysms on magnetic resonance vessel wall imaging. J Neurosurg 1-7, 2018

47) Shimonaga K, Matsushige T, Ishii D, et al.: Clinicopathological insights from vessel wall imaging of unruptured intracranial aneurysms. Stroke 49: 2516-2519, 2018

48) Larsen N, von der Brelie C, Trick D, et al.: Vessel wall enhancement in unruptured intracranial aneurysms: an indicator for higher risk of rupture? high-resolution MR imaging and correlated histologic findings. AJNR Am J Neuroradiol 39: 1617-1621, 2018

Address reprint requests to: Tomohiro Aoki, MD, $\mathrm{PhD}$, Department of Molecular Pharmacology, Research Institute, National Cerebral and Cardiovascular Center, 5-7-1 Fujishiro-dai, Suita, Osaka 565-8565, Japan. e-mail: tomoaoki@ncvc.go.jp 